bande de plomb (antimonié de préférence), placée en dehors de l'électrolyte. On peut couler cette tête de plomb autour des crayons, sur une longueur de 10 à $20 \mathrm{~mm}$, à l'aide d'un moule spécial ; on peut aussi visser les tig'es dans la masse de plomì. On laisse entre chaque crayon une distance de 5 a $10 \mathrm{~mm}$. environ.

Les avantages résultant de l'emploi de l'aluminium sous cette forme sont les suivants : Circulation de l'électrolyte pendant le fonctionnement, permettant autant que possible légalisalion de la température; attaque aussi réduite et aussi régulière que possible, due à l'état physique de l'aluminium étiré (propriété analogue à celle des crayons de zinc étiré dans les piles Leclanchẻ); remplacement aisé ét peu coûteux de l'électrode-aluminium lorsque, par suite du fonctionnement, cette dernière se trouve détériorée.

Liusure de l'aluminium, dans les soupapes électrolytiques, constitue la dépense la plus importante ; sous forme de tiges élirées, le prix de l'aluminium n'est que de très peu supérieur au prix du métal brut.

On peut employer tous les électrolylos habituels; toutefois, on recommande, comme rentrant dans le cadre de cette invention, l'usage des solutions de borates alcalins, et plus particulièrement des borates d'ammoniaque. Ces derniers se préparent trés simplement, sur place, en dissolvant lacide borique du commerce dans de leau ammoniacale. Cet electrolyte fonctionne bien, et son prix de revient est très raible.

En résumé, la soupape électrolylique Limb est caractérisee par l'emploi de l'aluminium sous forme de "crayons" étirés a la flière, absolument comme les crayons de zinc des piles Leclance, et par l'usage, comme électrolyte, des solutions de borates alcalins, particulièrement des borates d'ammoniaque.

E. C.

\section{SÉPARATION DES PUISSANCES RÉELLLE ET MAGNÉtIQUUE dans les Calculs relatifs aux Courants alternatifs}

La méthode repose sur la conservation de la puissance magnétisante et de la puissance réelle, Je dois dire ici que la démonstration que j'ai donnée de ce fait au Congrès de 1900 n'est pas absolument générale; jen donne depuis une démonstration générale dans les conférences que je fais tous les ans à l'Ecole supérieure d'Electricité, mais il me paraît de peu d'intérêt de reproduire ici cette démonstration. Il suffit de se rappeler que les puissances réelles s'ajoutent algébriquement, qu'il en est de même des puissances magnétisantes, et qu'en chaque portion d'un réseau la puissance apparente est la racine carrée de la somme des carrés des puissances réelle et magnétisante.

Je pourrais prendre l'exemple banal d'un réseau quelconque, comportant des lampes, des moteurs, etc., mais, dans le but d'accrôitre l'intérêt de la question pour le lecteur, j'appliquerai la méthode à l'uñ des deux montages quej'ai indiqués en 1890 comme permettant

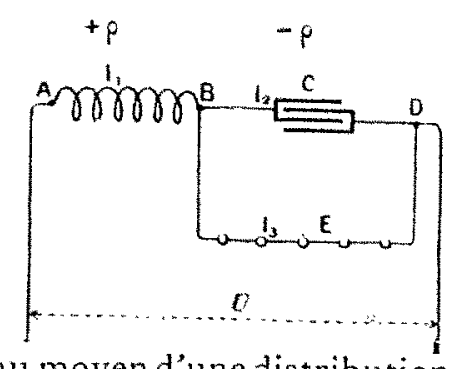
d'obtenir une intensité constante au moyen d'une distribution à tension constante, par exemple celui de la figure ci-jointe.

Entre $A$ et $B$ est une bobine de self-induction, de réactance $p$, que nous supposerons, pour simplifier, n'absorber aucune énergie. Entre $B$ et $D$ est une capacité $C$, que nous supposerons, également, n'absorber aucune énergie, et telle que sa réactance soit - $p$, d'où :

$$
\frac{\mathrm{I}}{{ }_{\omega} C}=p \quad \text { et } \quad \omega^{2} L C=\mathrm{I}
$$

Entre $B$ et $D$ se trouve, également dérivé, un circuit d'utilisation contenant n'importe quoi, pourvu que ce n'im. porte quoi n'introduise pas d'harmoniques sensibles dans la force électromotrice ou dans le courant.

Soient, dans ce circuit: $I_{3}$ l'intensité efficace; $p$ la puissance réelle; $m$ la puissance magnétisante; $E$ la différence de potentiel efficace aux bornes B et D.

Soit $I_{2}$ l'intensité efficace dans la capacité; $I_{1}$ l'intensité efficace dans la self-induction $\mathrm{AB} ; U$ la différence de potentiel efficace entre $\mathrm{A}$ et $\mathrm{D}$.

Dans la self-induction et dans la capacité, il n'y a pas de puissance réelle, et les puissances magnétisantes sont respectivement :

$$
p_{1}^{2} \quad \text { et } \quad-p I_{2}^{2}=-\frac{E^{2}}{p}
$$

L'application des prémisses conduit aux trois équations suivantes:

Pour le circuit d'utilisation considéré seul:

$$
p^{2}+m^{2}=E^{2} I_{3}^{2}
$$

Pour les deux circuits dérivés entre B et D:

$$
p^{2}+\left(m-\frac{E^{2}}{p}\right)^{2}=E^{2} I_{1}^{2}
$$

Pour l'ensemble:

$$
p^{2}+\left(m-\frac{E^{2}}{\rho}+\rho_{1}^{2}\right)^{2}=U^{2} I_{1}^{2}
$$

Développant (3) et combinant avec (2), il vient:

$$
U^{2}=E^{2}+\left(\rho I_{1}\right)^{2}+2 \rho\left(m-\frac{E^{2}}{\rho}\right)
$$

Développant (2), et combinant avec (1), il vient:

$$
p^{2} I_{4}^{2}=p^{2} I_{3}^{2}+E^{2}-2 m p
$$

Combinant ces deux dernières équations, il vient:

$$
U=p I_{3}
$$

On peut déduire immédiatement les courats $I_{2}$ et $I_{1}$, la tension aux bornes de la self-induction, etc., et ces résultats sont obtenus sans qu'il ait été besoin d'utiliser les équations aux valeurs instantanées.

En général, les calculs numériques concernant un réseau ordinaire sont encore plus simples: il suffit de cheminer de proche en proche des appareils d'utilisation jusqu'à la source, en faisant des additions, soustractions, multiplications, divisions, carrés et racines carrées.

Afin de pouvoir appliquer la méthode aux appareils doués d'induction mutuelle, et en passant de circuits-à d'autres circuits isolés des premiers, 11 faut démontrer qu'il $y$ a encore conservation des puissances dans ces conditions. Cela est facile, et je ne $\mathrm{m}^{\prime}$ attarderai pas non plus à faire ici cette démonstration, qui a été déjà faite par M. Nougier. (*).

La méthode permet ainsi d'utiliser ses qualités si pré-

(*) Nougrer. - Etude des installatio'ns à courants alternatifs par la méthode des grandeurs wattées et magnétisantes. (L'Eclairage Electrique, 1906). 
cjeuses au calcul des appareils à courants alternatifs : transformateurs, alternateurs, moteurs. Je me bornerai, aujourd'hui, à montrer comment la méthode peut être utilisée au calcul de la chute de tension dans un transformateur én charge, én tenant compte des fuites magnétiques, c'est-àdire subsituée aux méthodes graphiques de Kapp ou autres, permettant la solution de ce problème.

De quelque manière que le problème soit traité, il faut connaître les fuites magnétiques pour le primaire et pour le secondaire. Ces fuites connues, il est facile d'en déduire les forces contre-électromotrices développées par elles dans les circuits, primaire et secondaire, forces contre-électromotrices qui sont en quadrature avec les courants. Dans les méthodes graphiques auxquelles je viens de faire allusion, on introduit alors ces lorces contre-électromotrices par leurs phases et l'on procède à leur construction. Ici, il faut multiplier les forces contre-électromotrices par les courants respectifs pour en déduire les puissances magnétiques primaire et secondaire perdues dans l'appareil, indépendamment de celle perdue à la magnétisation du circuit magnétique commun aux deux circuits, et qui se calcule encore plus facilement.

Soient alors: $P_{2}$ la puissance réelle que débite le secondaire; $M_{2}$ la puissance magnétisante que débite le secondaire; $r_{2}$ la résistance du secondaire; $p_{2}$ la réactance de fuites du seçondaire calculée comme il vient d'être dit ( $I_{9}{ }^{2}=$ perte de puissance magnétisante secondaire); $I_{Q}$ le courant secondaire.

On a alors, pour la puissance réelle totale du secondaire,

$$
P_{2}^{\prime}=P_{2}+r_{2} I_{2}{ }^{2}
$$

et, pour la puissance magnétisante totale du secondaire :

$$
M_{\mathrm{q}}=M_{\mathrm{Q}}+\rho_{\mathrm{2}} I_{\mathrm{q}}{ }^{2}
$$

La puissance apparente totale du secondaire est donc:

$$
A_{2}=V \overline{P_{2}^{2}+M_{2}^{2}}
$$

La force électromotrice secondaire est alors :

$$
E_{2}^{\prime}=\frac{A_{9}}{I_{2}}
$$

Par le rapport du nombre des spires, il est facile de passer à la force électromotrice primaire $E_{1}^{\prime}$.

Si $p_{f}$ est la puissance réelle perdue dans le fer, $m_{f}$ la puissance magnétisante nécessaire à la formation du flux dans le fer du transformateur, on a le courant primaire :

$$
I_{1}=\frac{\sqrt{\left(P_{2}^{\prime}+p_{f}\right)^{2}+\left(M_{2}+m_{f}\right)^{2}}}{E_{1}^{\prime}}
$$

Si l'on a encore: $r_{i}$ résistance du primaire; $\rho_{1}$ réactance de fuites du primaire $\left(\hat{r}_{1} I_{1}{ }^{2}=\right.$ perte), la puissance réelle aux bornes du primaire est :

$$
P_{1}=P_{2}^{\prime}+p_{f}+r_{1} I_{1}^{2}
$$

la puissance magnétisante aux bornes du primaire est :

$$
M_{1}=M_{2}+m_{f}+p_{1} I_{1}^{2}
$$

De sorte que la puissance apparente aux bornes du primaire est :

$$
A_{1}=\sqrt{P_{1}^{2}+M_{1}^{2}}
$$

et la tension aux bornes du primaire:

$$
E_{1}=\frac{A_{1}}{I_{1}}
$$

Ce calcul, fait une fois en donnant a $P_{2}$ et $M_{2}$ des valecirs nulles, une autre fois en donnant a $P_{Q}$ et $M_{2}$ leurs valeurs respectives en charge, donne deux valeurs de $E_{1}$ : celles que $E_{1}$ devrait avoir à vide et en charge pour que $E_{\mathrm{g}}$ soit invariable. Le rapport de ces deux valeurs diffère extrêmement peu du rapport entre les différences de potentiel aux bornes du secondaire à vide et en charge; il lui est pratiquement égal.

(La Renue Electrique.)

P. Boucherot.

\section{LE MOIS HYDRO-ÉLECTRIQUE}

\section{ACADEMIE DES SCIENCES}

\section{MÉCANIQUE ET ÉLECTRICITÉ}

Sur le rôle et la nature de la décharge initiale (trait de feu dans l'étincelle électrique. - Note de M. G.-A. Hemsalech, Séance du 25 mars 1907 .

L'augmentation de la self-induction du circuit de décharge d'un condensateur est accompagnée de changements très marqués dans l'aspect et le spectie d'une étincelle placée dans ce circuit (I). A partir d'une certaine valeur de la self-induction, les raies de l'air, dues à la décharge initiale, disparaissent complètement.

Sans self-induction, le trait de feu est très lumineux par rapport aux observations qui suivent, et une grande partie de l'énergie mise en jeu dans la décharge, est nécessaire pour sa production. [D'intervalle entre la décharge initiale et la première oscillation est toujours un peu plus grand que les intervalles entre les oscillations mêmes, comme l'a monfré M. Tissot (2). L'amortissement des oscillations est considérable, et l'on ne peut que difficilement obtenir plus qu'une dizaine dans une même décharge:
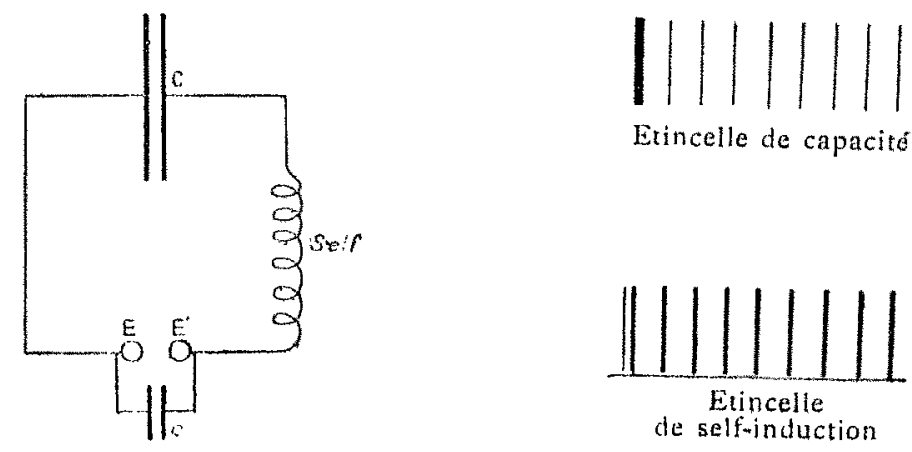

Etincelle de capacité

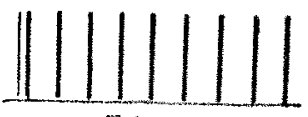

Etincelle de selfinduction

Mais, si, en augmentant la self-induction, on dépasse un certain point critique, le caractère et la décharge initiale change brusquement : elle devient trés faible, et une quantité minime d'énergie suffit pour sa production, de sorte que presque toute l'énergie de la décharge du condensateur est mise à profit des oscillations.

Ces dernicres deviennent alors très énergiques; l'amortissement est beaucoup moins grand que dans le cas précédent, et l'on peut facilement obtenir de 30 à 40 oscillations dans une même décharge. L'intervalle qui sépare la décharge initiale de la première oscillation est maintenant infiniment plus petit que l'intervalle entre deux oscillations successives, et, si l'on continue a augmenter la self-induction, cet intervalle, ainsi que l'éclat de la décharge initiale, restent constants, et seules les oscillations sont infuencées. Lintervalle entre la décharge initiale et la première oscillation est de moins de $10^{-5}$ secondes.

En me servant de la méthode photographique (pellicule mobile) et de celle du courant d'air (3), jai constaté que la valeur de la self-

(1) Hemsalech, Comptes-rendus, t. CXXIX, 1809, p. 285 et t, CXXXII, $1901 ;$ p. 917

(2) Tissot, Thèse de doct. Paris, 19o5, p. 77-80.

(3) Comptes-rendus, t. CXI, igo5, p. 1103. 